\title{
A Novel Group Mobility Model for Software Defined Future Mobile Networks
}

\section{Sureshkumar A ( $\nabla$ sureshkrishnaphd@gmail.com )}

Sri Krishna College of Technology

\section{Surendran D}

KPR Institute of Engineering and Technology

\section{Research Article}

Keywords: Group mobility management (GMM), Software defined network (SDN), Future Mobile Networks, MoMo model, handover

Posted Date: June 2nd, 2021

DOI: https://doi.org/10.21203/rs.3.rs-474298/v1

License: (1) This work is licensed under a Creative Commons Attribution 4.0 International License. Read Full License 


\title{
A Novel Group Mobility Model for Software Defined Future Mobile Networks
}

\author{
${ }^{1}$ Mr.A.Sureshkumar, ${ }^{2}$ Dr.D.Surendran \\ ${ }^{1}$ Assistant Professor, Department of Information Technology, Sri krishna College of Technology, Coimbatore, \\ Tamilnadu, India.E-mail : sureshkrishnaphd@gmail.com \\ ${ }^{2}$ Professor, Department of Computer Science and Engineering, KPR Institute of Engineering and Technology. \\ Coimbatore, Tamilnadu, India.
}

\begin{abstract}
Nowadays, a massive amount of data leads to cause network traffic and inflexible mobility in future mobile networks. A new Group Mobility Model (GMM) named MoMo is introduced that addresses the issue of the aforementioned problems. Even though, software defined network $(\mathrm{SDN})$ is functional with network-rooted mobility protocols that enhance the network efficiency. Some existing network-rooted mobility administration methods still undergo handover delay, packet loss, and high signaling cost through handover processing. In this research work, SDN-based fast handover for GMM is proposed. Here, the neighbor number of evolving node transition probabilities of the mobile node (MN) and their obtainable resource probabilities are estimated. This makes a mathematical framework to decide the preeminent number of the evolving nodes and then allot these to mobile nodes virtually with all associations finished by the exploit of Open-Flow tables. The performance examination demonstrates that the proposed SDN rooted GMM technique has the enhanced performance than the conventional handover process and further technique by handover latency, signaling cost, network throughput, and packet loss.
\end{abstract}

Keywords: Group mobility management (GMM), Software defined network (SDN), Future Mobile Networks, MoMo model, handover.

\section{Introduction}

According to the statistics of mobile technology, an extensive increase has been made in the past 20 years and it is continued drastically in the near future [1]. Nowadays, the mobile communication network handles immense amount of data which leads to cause network traffic. Thus the network uses advanced technologies such as Artificial Intelligent, Internet of Things, and Software Defined Network (SDN), which eagerly support the network bandwidth [2].In future mobile network, mobile video traffic is the main factor that should be compensated by virtual architecture. This infrastructure is based on Software Defined Network which provides flexible and on-demand service to the future 5G/6G mobile network. A software defined networking (SDN) is a programmable network architecture composed of three layers, namely, infrastructure, control, and application layer, respectively. Open flow is a bidirectional link which is used to direct the signalling message between the underlying network planes and SDN controller. Higher flexibility, better resource allocation, and improved performance are the potential benefits that should be governed by SDN [3]. In $5 \mathrm{G} / 6 \mathrm{G}$, the dependency of SDN's physical network is being reduced to generate high Quality of Service (QoS). 
Ultra dense network, a dense coverage model which supports high bandwidth in future mobile network (5G/6G) [4-6]. However, the dense network is not suitably able to perform the handover process using the conventional mobility model. Thus the network is required to change the mobility model to enhance the network performance. This effectiveness not only improves the handover process but also maintains high on-demand resource allocation and better QoS. To improve handover efficiency, mobility model selection plays an essential role in it. A Beam forming concept is a flexible operation meant to increase the available resources usage to sustain QoS [7].

However, the aforementioned technologies such as SDN and ultra-dense network perform data forwarding task with centralized mobility management (CMM), i.e., mobile anchor (MA) for home agent and local mobile anchor (LMA) for network routing respectively [8]. It is a central agent, that serves to sustain MN locations and redirect the traffic to them. CMM approaches handles some obstacles such as low scalability, high overhead, single node failure, etc. The following issue have been resolved using a new paradigm named as Distribution Mobility Management (DMM) [9]. DMM is a distributed through the mobility agent to overcome the single node failure because if a mobility agent is failed to perform their job, then the other mobility agent within the network will take over the job of the failed node. This arrangement is being reduced the mobile data traffic by improving the handover delay, scalability, etc. More investigation was performed to confirm the SDN based DMM technology and it is noted in several literatures [10-12].

In a general viewpoint, the mobility model is categorized as individual and group mobility model [13]. These two agents are continuously making a handover service to mobile nodes $(\mathrm{MN})$. Group mobility model (GMM) is a mobility pattern described to predict the movement of the mobile node in terms of continuous changeover time such as velocity, location, and acceleration [14]. In this model, each of the mobile nodes moves randomly together within the group using Random Waypoint approach [15] and Reference Point Group Mobility Pattern (RPGP) [16].Both group mobility and individual mobility are used DynnaMo approach which provides a memory related model. The MoMo model combines memory-based individual model and flexible group model which increases the accuracy of the mobility model [17]. In this paper, we proposed software defined network based MoMo model for future mobile network to reduce the handover latency, signaling cost, and packet drop ratio.

The contribution of the proposed SDN based GMM work comprises of:

- The proposed model incorporates the mobility management module (MME) and admission control module. The MME includes the evolving node (EN) transition probability estimation and evolving node selection engines which are in the handover preparation phase.

- In the handover preparation phase, EN transition is computed for each neighbor node so that EN_ID is updated periodically. Therefore, the estimated EN_ID is transferred to the Open Flow table of the mobile node. If the current EN duration is expired, then automatically the MN node checks the optical flow (OF) table and chooses the next 
evolving node. The target EN influence OF table and it can find that the respective MN_ID is included in this table, then it will send handover acknowledgement to the MN. This concludes that both EN and MN nodes start preparing access to exchange their messages.

- In the handover phase, the grouping of each node is checked periodically based on two condition, namely, free state and forced state process.

- Finally, the handover performance such as handover latency, throughput, signaling cost, and packet loss are assessed for the proposed work which shows outperformed efficiency than the exiting GMM technique.

The association of this work is offered as pursues: Section 2 explains the literature review on recent SDN based mobility models. Section 3 describes the working of the proposed SDN based handover technique for future mobile networks. Section 4 portrays the simulation results and discussions. Lastly, section 5 ends the paper chased by the references.

\section{Related Work for Research}

Several existing works of mobility model with its issues, improvisation and challenges were discussed in this section.

Chung-Ming Huang et al. [18] presented a Bursty Multi-node Handover using partially DMM (BMH-DMM) which uses a control scheme to tackle the handover problem. The proposed technique applies three procedures, namely, choosing MAAR candidate, handover preparation, and handover phase to simultaneously exchanging the query message to destination. Therefore, the approach uses a set of control schemes to minimize the handover delay and redundant message in Layers 2 and 3.In phase 1, Link_Going_Down (LGD) event is initiated from the set of mobile nodes (MN) which directs their message to MAAR1. After receiving the message, MAAR1 precisely needs to select proper destination among $M A A R_{i}$. Thus, MAAR1 derive score value for each $M A A R_{i}$ by using scoring function. After identified the score function from $M A A R_{i}$, MAAR1 establish a handover preparation to MAAR2. Finally, MAAR2 registers the CMD and handover the packet to the destination using a bidirectional tunnel. The benefit of this framework is to reduce the handover delay, packet loss, and signaling bandwidth but suffer from data integrity problem.

Luca Cominardi et al. [19] presented a more realistic network named as SDN with DNN technique. Packet delivery route is being optimized by reconfigured VLAN which shows a lack of scalability. Therefore, a new packet delivery procedure introduced by Sunghong Wei [20] processed through a soft anchor. It becomes more reasonable when combining the SDN technology with hybrid DMM method. Possibly, the approach of SDN based hybrid DMM (S-hDMM) is very effective to minimize high signaling cost. It performs two modes of procedure, namely, initial registration and handover. In registration procedure, the Optical Flow Switch (OFS) start receives the MN's message and forwards to S-hDMM. The controller creates the binding catch entries to update the MN's IP address to the OFS table. After the ended registration, the handover process occurs. Choosing the best anchor is 
an important factor which reduces packet loss during training. OFS, a soft anchor is the best choice to reconfigure the packet delivery route for higher efficiency.

Battulga, Davaa, sambuu et al. [21] developed a distributed mobility model for selecting a mobility anchor (MA). The paper implemented some factors to select the mobility anchor based on cost function. Handover procedure provides the serving and target cells to transfer context information in which the handover commands return back to the subscriber at the end. Indispensible to note that the respective model is much effective to enhance the load balancing, packet delivery cost, and proper handover procedure but suffer from signaling performance.

Yong-hwan Kim et al. [22] presented a software-designed network (SDN) based on DMM for improving the LTE/EPC network performance. In conventional LTE/EPC networks, improper separation of data and control plane functionality may degrade the handover latency and radio resource allocation in Inter-technology. These issues are being resolved by the proposed architecture in the way of distributing the data plane through the gateway closer to the UEs, a centralized control plane virtually, and by clearly separating the control plane and data plane. The following procedure has to solve the latency problem, but the architecture becomes more complex than the other network because the system is virtually in-built. Another approach of the newly upgraded model named group mobility tolerates a valuable solution to the data exchanging mechanism. Cherry Ye Aung et al. [23] systematically reviewed a GMM by designing an accurate mobile ad hoc network (MANET). In this scenario, the author focuses on the categories of grouping model. Based on the movement of group members, the model is classified into four classes which independently control the location of an adjacent regions while moving. The review paper explains the movement of each group and their design features. Vehicular cognitive radio node is an application oriented task utilized to make secure communication based on group mobility management (GMM) and is developed by Mani Shekhar Gupta et al. [24]. Potentially, the model chooses an improved network resource to achieve high throughput. Nevertheless, the network occupies congestion while performing mobility.

\section{Proposed Methodology}

With the elevated challenging and the rising of mobile users (MU), the mobile network undergoes several issues such as handover (HO), data traffic, network routing, reliability, scalability, network signaling etc. To gather the inclination of increasing MUs, a novel group-based mobility management method is proposed to resolve the most challenging $\mathrm{HO}$ issue. Here, the particulars of the proposed SDN-based Fast HO control technique for GMM (SDN-GMM) are presented. A novel Group based mobility management (GMM) method, named MoMo is proposed by utilizing distributed mobility management (DMM). Different from the existing methods, the backward fast HO method is employed, which permits SDN to have an elevated possibility to end the HO training processing prior to detaching from the present sub-network, to diminish the packet loss rate and $\mathrm{HO}$ latency. The proposed novel GMM has three phases of operation namely Initial Phase, HO Preparation phase and HO Phase. 


\subsection{Initial Phase}

The MN is still associated with previous access points (AP) that detect the next AP. It established a signal strength which is advanced than the predefined threshold and is the uppermost one amid the sensed APs; then simultaneously the MNS threw the report message enclosing the next AP information to the initial one.

\subsection{Handover Preparation phase}

This phase comprises of two modules such as the mobility management module and admission control module. This phase administers the dummy small cells and MNs in the data plane for mobility management. Furthermore, the mobility management entity (MME) is made to handle the HO procedure. In this way, the required mobility related data, for instance, the MN subscription data, mobile identification, and tackling trail area posts are attained. The mobile node id (MN_ID) and Evolving Node id (EN_ID) parameters are employed for MNs and small cells [25].In the proposed SDN -GMM, MoMo model, the number of evolving nodes in the network contains a hexagonal architecture. This utilizes the automatic neighbor relation (ANR) function of evolving node count by which the neighbor relation tables are updated. Accordingly, the proposed SDN-GMM MoMo model reaches the valid neighbor relations of the evolving new nodes from these tables. Figure 1 demonstrates the proposed network architecture.

After calculating the transition possibilities for the neighbor evolving nodes, an obtainable resource probability of these neighbor evolving nodes are projected. According to the outcomes of this procedure, the next evolving node are estimated and allotted virtually to the MN before the movement. Thus, the predicted EN_ID is moved to the Open Flow (OF) table of the MN. Moreover, the time for this evolving node is computed and given to the value OF table of the MN. Every aforesaid method is performed for all evolving nodes situated on the MN movement path. 
If the time of the present evolving node closes, then $\mathrm{MN}$ views OF table to estimate the next evolving node. Accordingly, as revealed in Fig. 1, the MN propels a HO appeal to the identified target evolving node. The contact amid the $\mathrm{MN}$ and target evolving nodes is performed on the random access channel (RACH). In future mobile networks, the RACH is

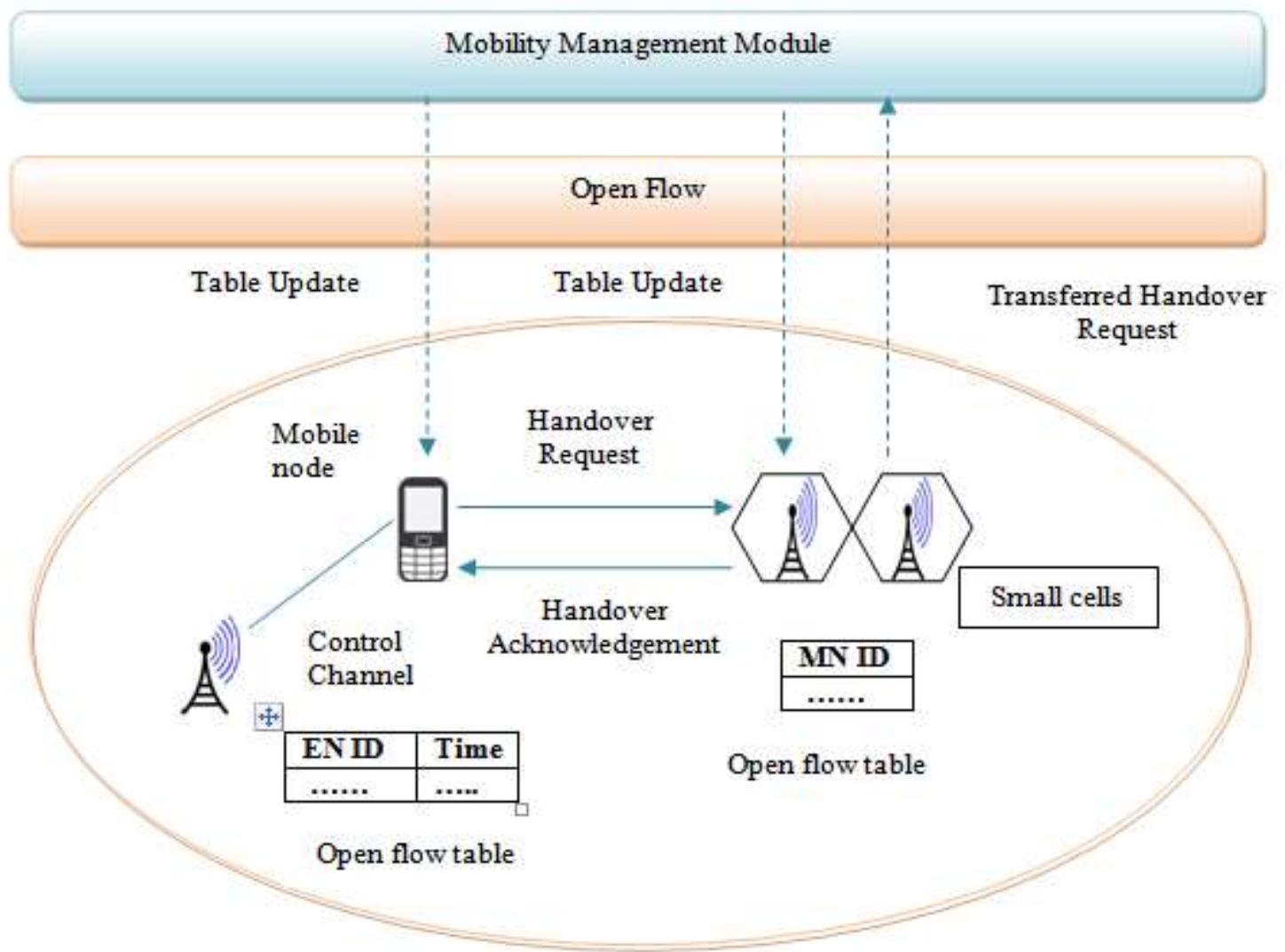

utilized by $\mathrm{MN}$ to start the session with an arbitrary access preamble throughout the primary movement of the connect process. In addition, this preamble comprises the MN_ID. Next, goal evolving node manages OF table to discover this arriving MN_ID. If this MN_ID is integrated in the table, $\mathrm{HO}$ response is given to the MN. This response specifies that the HO appeal is established by the evolving node. Then the connect process continues amid the MN and evolving nodes with the equivalent message series as downlink shared channel (DL$\mathrm{SCH}$ ), uplink shared channel (UL-SCH). Here, the delays observed in the HO training stage are investigated. If the MN_ID is not identified, this request is transferred to the controller. The controller updates the set OF tables accordingly.

\section{Figure 1: The proposed network architecture}

\subsection{Handover Phase}

The HO phase is followed by the HO preparation phase. This phase describes the connectivity steps of the nodes in the SDN based intelligent future network. The proposed SDN-GMM based MoMo model defines the binding conditions related tophysical proximity between the nodes. The binding condition between two nodes namely $i$ and $j$ are in same group, are referred to as group mates, and is defined as in (1) 


$$
d_{i j} \leq D_{c}
$$

Where, $d_{i j}=d_{j i}$ is the distance among nodesiandj. Ifthe binding condition in Eq. (1) is satisfied, the two nodes are said to be distance $D_{c}$ connected. Consider a group of size M. For the generic node $j$ the set of $M_{j}^{c}$ group mates that the node detects as connected is called its connected set. The ratio between $M_{j}^{c}$ and the total number $\mathrm{M}$ of group mates is called grouping factor $\rho j$ in (2):

$$
\rho j=\frac{M_{j}^{c}}{M-1}
$$

The behavior of node $j$ depends on the grouping condition (GC) defined on $\rho j$ in (3):

$$
\rho j \geq \rho_{\min }
$$

where the grouping threshold $\rho_{\min }$ is a tunable model parameter. Every node occasionally verifies whether the GC is satisfied, with period $\nabla u$ based on the result, the node penetrates in anyone of the two subsequent states like free and forced:

- Free State occurs when the GC is satisfied. Here the node freely moves based on the boundless mobility model;

- Forced state occurs when the GC is not satisfied. Here the node travels towards the closest group mate, $\mathrm{k}$, is not part of its connected set, to improve its grouping factor. The speed variables $v$ and $\theta$ are set as in (4) and (5):

$$
\begin{gathered}
v=v_{\max } \\
\theta=\left\{\begin{array}{l}
\min \left(\beta_{k j}, \theta_{\text {old }}+\gamma_{\min } T_{l u}\right), \text { if } \beta_{k j} \geq \theta_{\text {old }} \\
\max \left(\beta_{k j}, \theta_{\text {old }}-\gamma_{\max } T_{l u}\right), \text { otherwise }
\end{array}\right.
\end{gathered}
$$

Where, $\beta_{k j}=\arctan \left(\frac{y_{k}-y_{j}}{x_{k}-x_{j}}\right),\left(x_{k}, y_{k}\right)$ and $\left(x_{j}, y_{j}\right)$ are the locations of nodes $\mathrm{k}$ and $\mathrm{j}$, respectively, $\theta_{o l d}$ is the previous direction, and $T_{l u}$ is the time elapsed while the final position update1. Equations (4) and (5) ensure that node $j$ attains the chosen group mate $\mathrm{k}$ in the shortest probable time frame, while evading although, destruction of the limitation on linear and angular speed.

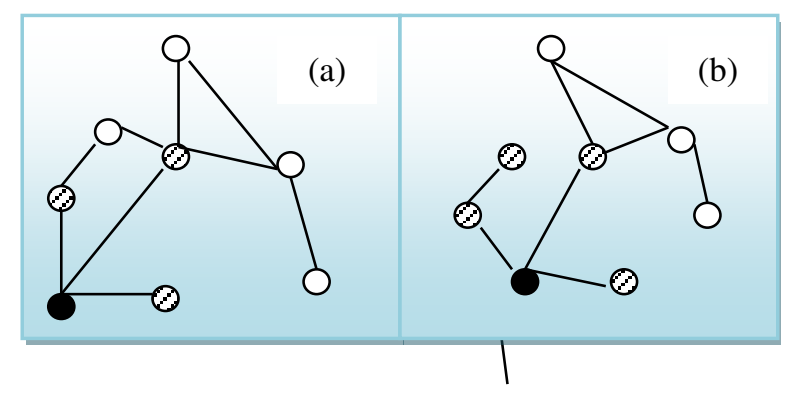




\section{Figure 2: Example of the function of the SDN - GMM MoMo mobility model for a group of 8 nodes.}

In Fig 2 a), the measured node (black node) is linked to three striped nodes in its collection, out of its 7 group mates, and the grouping factor $\rho=3 / 7$ calculated by the node is lesser than the necessary $\rho_{\min }$. As a result, the node goes in forced mode and travels towards the nearby group mate among its connected set. The node preserves this behavior until the GCis is satisfied, evolving towards the situation in 2. b), where the range of the connected set is increased to 4 , corresponding to $\rho \geq \rho_{\min }$.

The behavior defined in the forced mode, that is, moving towards the closest group mate not in the connected set, is not the only possible one. More complex behaviors, e.g., moving towards the centroid of the points of group mates, can be easily established in the framework of the proposed SDN -GMM MoMo model. Figure 2demonstrate an instance of the application of the SDN -GMM MoMo model in the case of a group of 8 number of nodes with $\rho_{\min }=0.5$. Lines between nodes indicate connectivity for the SDN - GMM MoMo mobility model. In Figure 2a), the size 3 of the connected set for the black node leads to a grouping factor $\rho=0.43$. The GC is thus not satisfied, and the node moves to the closest group mate not part of its connected set, until the condition is satisfied as in the configuration shown in Figure 2b, in which $M_{c}=4, \rho=0.57$ ).

The definition of connectivity, and the corresponding meaning of the threshold $D_{c}$, is a key feature in SDN -GMM MoMo. The replicate for a flexible definition of the theory of associated, rooted on the application scenario:

- connectivity related to radio communications - here two nodes are associated either during a straight radio connection (physical layer associativity), while they are in the radio range, or during conveying, assured by additional group mates (network associativity);

- Connectivity is rooted on a radio-independent constraint - for instance, if a collection will become a security team, material visibility may correspond to association: a team member will go freely until it is capable to view a minimum count of team members, and travels nearer to the extra members of the team when the circumstance is not met any longer.

\section{Simulation Results and Discussions}

In this section, the HO presentation is evaluated with the proposed SDN- GMM model and estimates performance metrics including signaling cost, average HO latency, and packet loss rate. The simulation result of the proposed method is estimated by using NS-3 network simulator version 3.26. It is a discrete-event network simulator using the Open Flow module. By using it, the $\mathrm{HO}$ process working is verified. The simulation environment comprises of four sorts of network essentials with $50 \mathrm{MUs}, 10$ routers that manage 10 APs, 10 corresponding nodes, and $802.11 \mathrm{n}$ infrastructure. The simulation is continuously performed for 30 times to discover the regular outcomes. 


\subsection{Signaling cost}

The signaling cost is described as the HO mobility binding update overhead acquired throughout the HO processing. Hence, the signaling message delivery cost of the mobility management protocol is reliant on the result of the count of network hops, the dimension of a signaling message, and the weighting aspect in a wired and wireless network. In the proposed SDN-GMM method, the worldwide assessment allows SDN Controller to gain the agent router and manage signaling messages in MNs' HO processing. The MME and the MODULE are termed to hold mobility administration and handle MNs' registrations for having the GMM service. Thus, the signaling cost of the SDN-GMM technique is articulated as in (6)

$$
\text { cost }=\mu\left[\begin{array}{c}
h L_{\text {inform }} \text { hop }_{M N-\text { router }} \\
+\rho L_{\text {hi }} \text { hop } \\
+\rho L_{\text {hack }} \text { hop } \\
\text { router-router }
\end{array}\right]
$$

Where $h$ and $\rho$ are wired and wireless link's weight factor, $\mu$ is the arrival rate of each MN via router,Linform refers to the dimension of handover message,Lhi and Lhacks indicates the dimension of $\mathrm{HI}$ and Hack message respectively, hop specifies the connection between two nodes.

An advantage of the proposed SDN-GMM process is the decrease of the control packets for MN's andover processing. Figures 3 and 4portray the difference of the signaling cost rooted on diverse MN's velocities and different counts of hop counts. When the MN velocity is improved from 5 to $30 \mathrm{~m} / \mathrm{s}$ and the count of hop count is augmented from 1 to 10 . The count, MN velocity, and radius of cells of necessary binding update messages by the proposed SDN-GMM method is less than that of the traditional approach such as CMM[26], S-hDMM [20] and GMM [27]. The proposed technique achieves 187, 243, 300, 357, 419, and 498 signalling costs per packet versus $\mathrm{m}$ n's velocity from 5 to $30 \mathrm{~m} / \mathrm{s}$. Similarly, considering the signalling cost versus hop count and it shows that the proposed method attains $250,272,295,309,332,340,375,391,427$, and 439signalling cost per packet from 1 to 10 hop counts respectively.

The result of radius of the cell is pursued: the larger radius indicates the lower HO occurrence rate. Thus, the necessary binding update message decrease when the cell's radius enhances. Although the cell's radius is improved from 100 to $350 \mathrm{~m}$, the count of signaling messages of the proposed SDN-GMM technique is also lower than the existingtechniques. In this analysis result, the proposed SDN-GMM model attains an improved output of 990, 442, $384,216,206$, and 185signalling cost efficiency for 100, 150, 200, 250, 300 and 350 radius 
of cell in metres respectively. The comparisongraph of handover signaling loss versus radius of cell using different techniques is revealed in Figure 5.

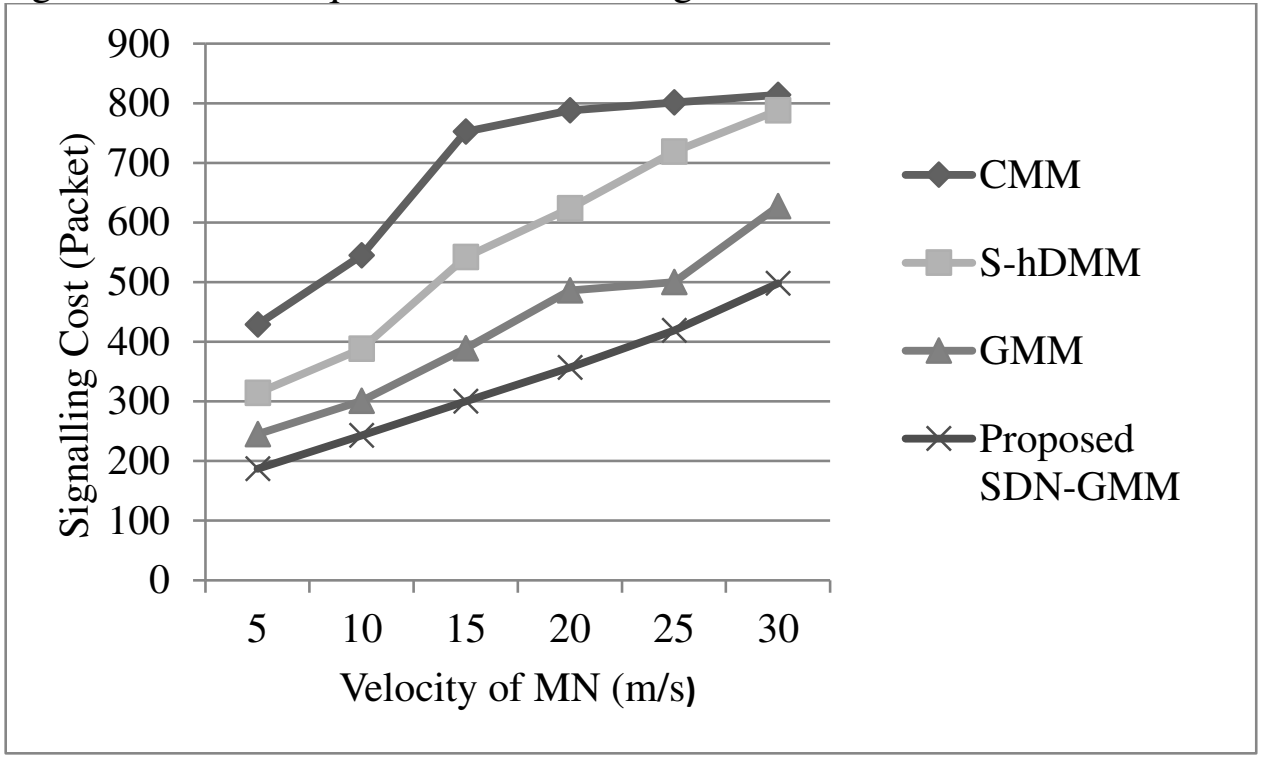

Fig. 3 Handover signaling cost versus velocity of MNs

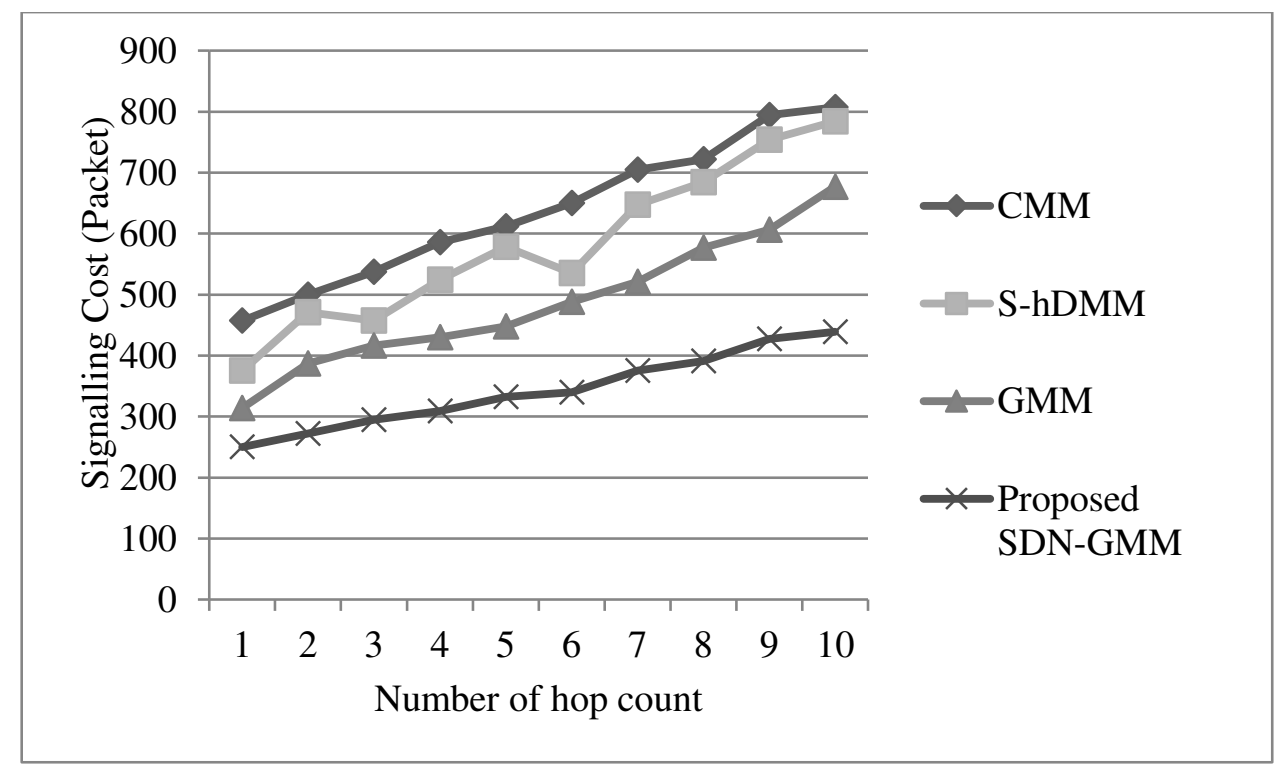

Fig. 4 Handover signaling cost versus number of hop count 


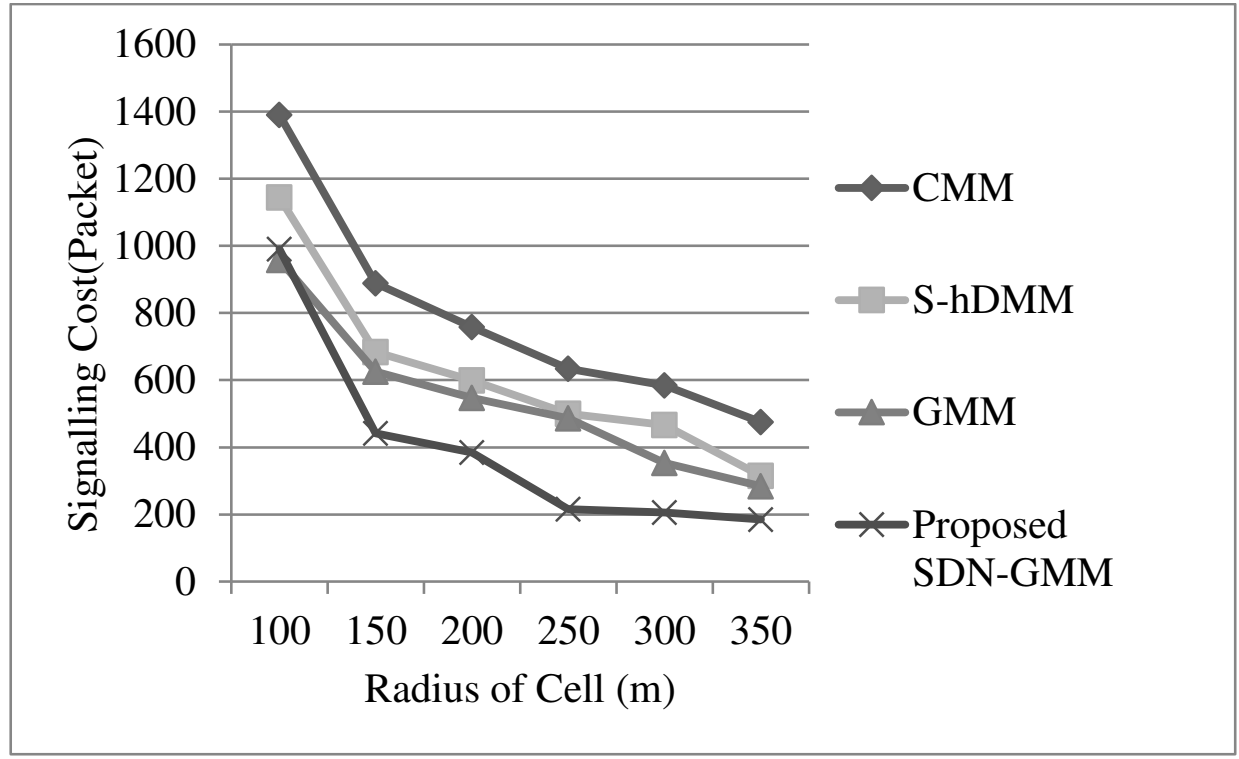

Fig. 5 Handover signaling cost versus radius of cells

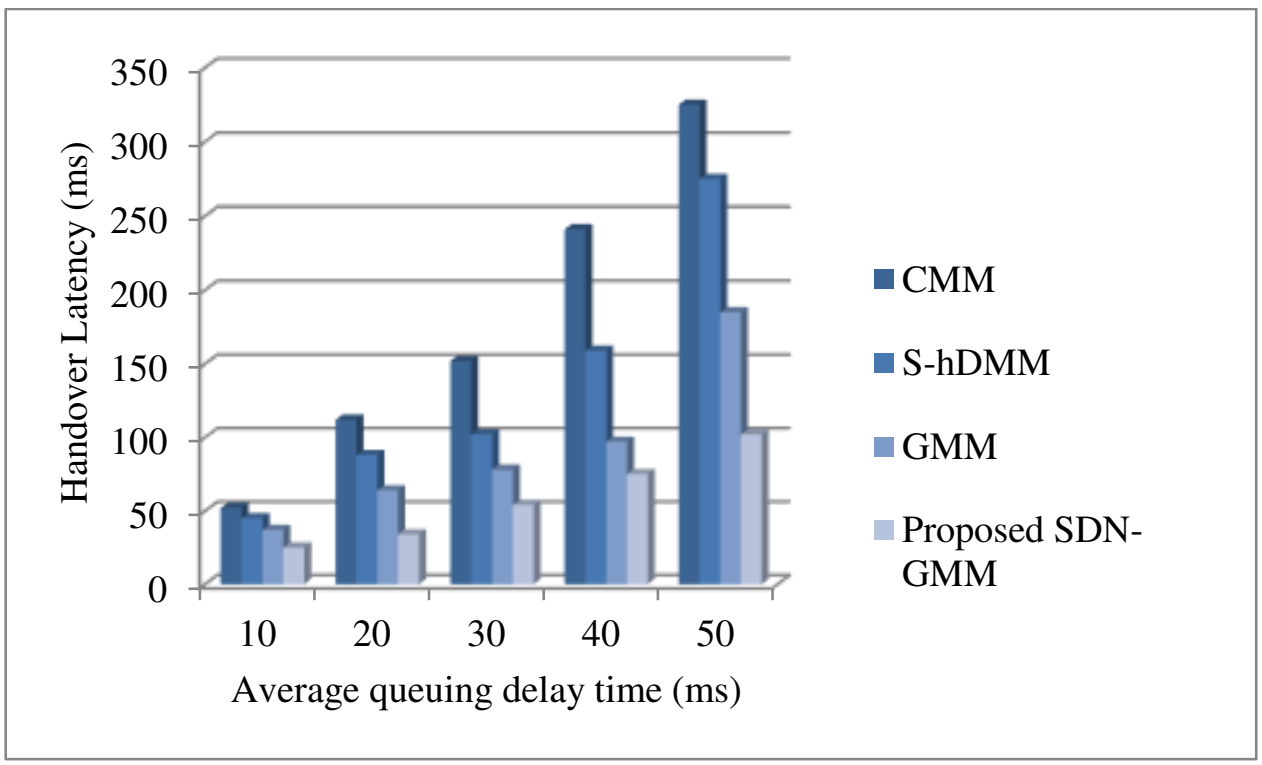

Fig. 6 Handover delay versus average queuing delay

\subsection{Handover latency}

The further metric for mobility management protocols is HO latency. The HO latency is described as the delay from the time when MN begins the HO process to the time when it accepts the first packet from its novel mobility router. The delay time is required to drive a packet over wired and wireless links and it contains propagation time, transmission delay, and queuing delay. The HO delay relies on the hop-count distance from the source to the destination. It is tacit that the wired links are steady and reliable. Then, the delay time of a packet of size $\mathrm{p}$ sent from $\mathrm{u}$ to $\mathrm{v}$ is represented as in (7):

$$
D t_{m, n}=\text { hopcount } *\left(\frac{S_{m s g}}{B W_{b n d}}+L_{p l}+W_{a q}\right)(7)
$$


Where $S_{m s g}, L_{p l}$, hop count, $B W_{b n d}$ and $W_{a q}$ are the average manage message volume, propagation latency, hop distance connectivity, the accessible bandwidth, and the average queuing delay at every router in wired links.

From Fig. 6, the proposed SDN-GMM method achieves 25, 34,54, 75, and 102 handover latency (ms) for average queuing delay time $10 \mathrm{~ms}, 20 \mathrm{~ms}, 30 \mathrm{~ms}, 40 \mathrm{~ms}$ and $50 \mathrm{~ms}$ respectively. It is clear that the average queuing delay at each router is amplified through the HO latency of the proposed SDN-GMM method which is lower than that of the traditional handover techniques.

\subsection{Throughput}

Throughput (thrt) is described as the whole volume of transmitted data packets in a session, which is $S_{a d} * L_{u}$ over the session delivery time and is formulated in (8).

$$
t h r t_{s d n-g m m}=S_{a d} * L_{u} /\left[\left(P D T_{s d n-g m m}+\left(S_{a d}-1\right) * \rho\right)+T_{H O-s d n-g m m} * t_{H O-n o}\right](8)
$$

Where, $S_{a d}$ is the average count of transmitted packets in a session, $L_{u}$ is the average packet size from a corresponding node to $\mathrm{MNs}$, and $\rho$ is the packet-to-packet gap time. $P D T_{s d n-g m m}$ is packet delivery time for transmitting $L_{u}$ packets. PDT is the packet delivery time from the corresponding node to MNs, which is calculated as the total delay time of transmitted packets given in formula (8). $T_{H O-s d n-g m m}$ is calculated as the ratio among the HO $\mu$ and the average session coming charge $\varphi$.

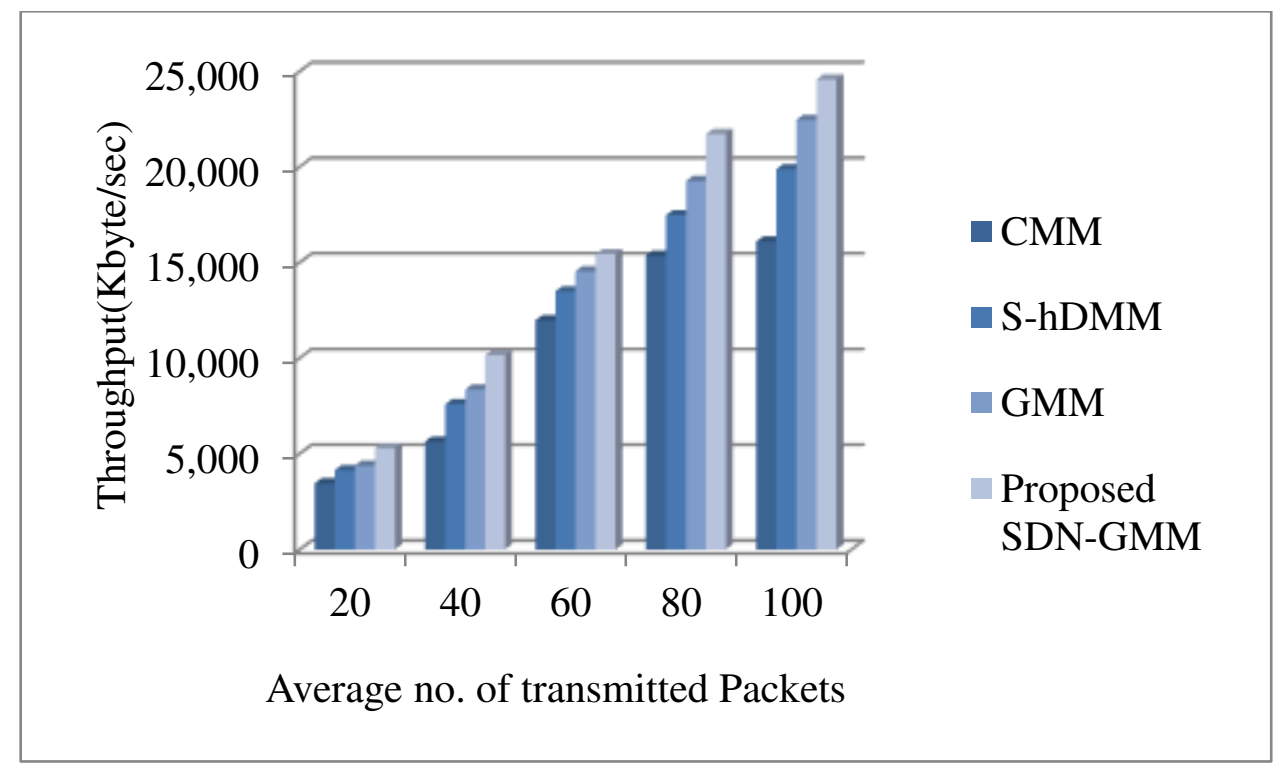

\section{Fig. 7 Throughput versus count of packets in a session}

Figure 7 shows the comparison of throughput performance between the proposed SDN-GMM technique and the existing techniques rooted on the count of packets. It shows the throughput of the proposed method is advanced than the existing methods when the average count of packets is improved from 20 to 100 packets. It shows that the proposed technique has high throughput and is listed as 5292, 10147, 15427, 21719, $24572 \mathrm{Kbyte} / \mathrm{sec}$ 
of throughput accomplished for 20,40, 60, 80 and 100 number of transmitted packets respectively.

\subsection{Packet loss}

When MN performs handover, it observes whether the packet loss can affect the HO procedure. MN's packet loss rate is proportional to either HO delay or session arrival rate $\varphi$ in unit of packet per second. Let Sad be the average count of delivered data packets during a session. Then, the packet loss rates of the use of the proposed SDN-GMM HO method are calculated in (9) as:

$$
C_{s d n-g m m}=\varphi * S_{a d} * T_{H O-d e l a y-s d n-g m m}
$$

Whereas $T_{H O-d e l a y-s d n-g m m}$ denote the average $\mathrm{HO}$ delay of the proposed SDN-GMM method.

Figure 8 depicts the packet loss situations of using the proposed SDN-GMM method and the traditional methods in terms of $\mathrm{HO}$ rates. Referring to Fig. 8, when the HO rate is improved from 0.12 to 0.28 , the count of missing packets by the proposed method is partly of that of that by the existing processes. It is experimentally proved that the total of lost packets by the proposed technique is smaller than that of the traditional techniques when the HO rate is improved. It is for the reason that the proposed SDN-GMM technique has the inferior HO latency time than the existing $\mathrm{HO}$ techniques and the momentary preestablished tunnel, which can be detached when all on-the-fly packets have been promoted to MN.

The proposed technique achieves 87, 91, 106, 175, and 192 packet loss amounts which are compared against the mobility rate of $0.12,0.16,0.20,0.24$, and 0.28 respectively.

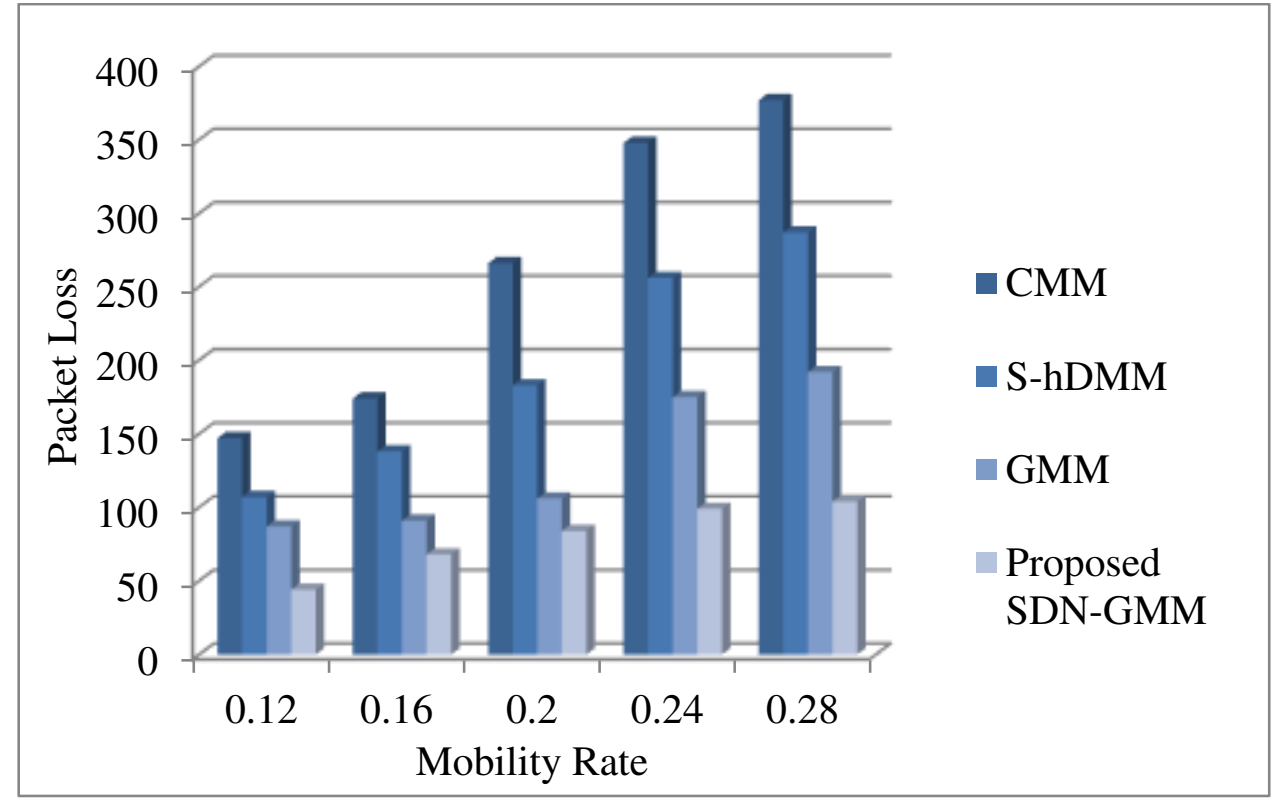

Fig. 8 Packet loss versus mobility rate 


\section{Conclusion}

In this work, an improved handover organized method for SDN -based fast handover for Group Mobility Model is proposed. The major contribution of the proposed SDN-GMM method are to allow $\mathrm{MN}$ be proficient to earlier finish the $\mathrm{HO}$ training processing and to attach to a new mobile router before $\mathrm{MN}$ disconnecting to the preceding mobile router. The packet loss of the proposed SDN-GMM method is condensed as of less HO latency than the existing GMM method and the handling of the temporarily established bidirectional tunnel, which can be detached when all on-the-fly packets have been forwarded. To prove the proposed method, it is compared with the traditional handover techniques over the NS-3 simulation environment. In future, the proposed SDN-GMM method can be practical in the vehicular setting.

Funding: There is no funding source.

Conflict of Interest: The authors declare that they have no conflict of interest.

\section{References}

[1] Mansoor Shafi, Andreas F. Molisch, Peter J. Smith, Thomas Haustein, Peiying Zhu, Prasan De Silva, Fredrik Tufvesson, Anass Benjebbour, and Gerhard Wunder, "5G: A tutorial overview of standards, trials, challenges, deployment, and practice", IEEE journal on selected areas in communications, vol.no.35, no. 6, pp. 1201-1221, 2017

[2] Catherine NayerTadros, Mohamed RM Rizk, and Bassem Mahmoud Mokhtar. "Software defined network-based management for enhanced 5G network services." IEEE Access vol.no.8, pp. 53997-54008, 2020

[3] Wenfeng Xia, Yonggang Wen, ChuanHengFoh, DusitNiyato, and HaiyongXie, "A survey on software-defined networking", IEEE Communications Surveys \& Tutorials, vol.no.17, no. 1, pp. 27-51, 2014.

[4] Peng Hao, Xiao Yan, Yu-NgokRuyue, and Yifei Yuan. "Ultra dense network: Challenges enabling technologies and new trends." China Communications, vol.no.13, no. 2, pp. 30-40, 2016

[5] Zhonglin Chen, Shanzhi Chen, Hui $\mathrm{Xu}$, and Bo Hu. "A security authentication scheme of 5G ultra-dense network based on block chain", IEEE Access, vol.no.6, pp. 55372-55379, 2018

[6] Xiaohu Ge, Song Tu, Guoqiang Mao, Cheng-Xiang Wang, and Tao Han. "5G ultradense cellular networks", IEEE Wireless Communications, vol.no.23, no. 1, pp. 7279, 2016

[7] SteliosTimotheou, IoannisKrikidis, Gan Zheng, and Bjorn Ottersten, "Beamforming for MISO interference channels with QoS and RF energy transfer", IEEE Transactions on Wireless Communications, vol.no.13, no. 5, pp. 2646-2658, 2014

[8] Javier Carmona-Murillo, Ignacio Soto, Francisco J. Rodríguez-Pérez, David CortésPolo, and José Luis González-Sánchez, "Performance evaluation of distributed mobility management protocols: Limitations and solutions for future mobile networks." Mobile Information Systems, 2017. 
[9] Yarisley Peña Llerena, Paulo RL Gondim, and Jaime Lloret, "Improving throughput in DMM with mobile assisted flow mobility," Transactions on Emerging Telecommunications Technologies, vol.no.29, no. 3, e3257, 2018.

[10] Michelle Perras, Juan Carlos Zuniga, Alexander Reznik, Carlos Jesus Bernardos, and Hao Jin, "Software defined networking distributed and dynamic mobility management" U.S. Patent No. 9,998,967, issued June 12, 2018.

[11] Jeong-A. Kim, Do Gun Park, and JongpilJeong, "Design and performance evaluation of cost-effective function-distributed mobility management scheme for softwaredefined smart factory networking", Journal of Ambient Intelligence and Humanized Computing, pp. 1-17, 2019

[12] Chung-Ming Huang, Duy-Tuan Dao, and Meng-Shu Chiang, "SDN-FHOR-DMM: a software defined network (SDN)-based fast handover with the optimal routing control method for distributed mobility management (DMM)", Telecommunication Systems, vol.no. 72, no. 2, pp. 157-177, 2019

[13] Tracy Camp, Jeff Boleng, and Vanessa Davies. "A survey of mobility models for ad hoc network research." Wireless communications and mobile computing, vol.no.2, no. 5, pp. 483-502, 2002

[14] Subodh Kumar, G. S. Agrawal, and Sudhir Kumar Sharma, "Impact of Mobility Models on MANETs Routing Protocols", INROADS-An International Journal of Jaipur National University, vol.no.3, no. 1, pp. 142-147, 2014

[15] Subodh Kumar, G. S. Agrawal, and Sudhir Kumar Sharma, "Impact of Mobility on MANETs Routing Protocols Using Group Mobility Model", International Journal of Wireless and Microwave Technologies,vol.no.7, no. 2, pp. 1-12, 2017

[16] Dayan AdionelGuimarães, EdielsonPrevatoFrigieri, and Lucas Jun Sakai. "Influence of node mobility, recharge, and path loss on the optimized lifetime of wireless rechargeable sensor networks." Ad Hoc Networks, vol.no.97, pp. 102025, 2020

[17] Luca De Nardis, and Maria-Gabriella Di Benedetto, "MoMo: a group mobility model for future generation mobile wireless networks", arXiv preprint, 1704.03065, 2017.

[18] Chung-Ming Huang, Duy-Tuan Dao, and Meng-Shu Chiang, "A Bursty Multi-node Handover scheme for mobile internet using the partially Distributed Mobility Management (BMH-DMM) architecture", Telecommunication Systems, vol.no. 69, no. 1, pp. 113-130, 2018

[19] Luca Cominardi, Fabio Giust, Carlos J. Bernardos, and Antonio De La Oliva, "Distributed mobility management solutions for next mobile network architectures", Computer Networks, vol.no.121, pp. 124-136, 2017

[20] SunghongWie, "SDN-based Hybrid Distributed Mobility Management", Journal of information and communication convergence engineering, vol.no .17, no. 2, pp. 97104, 2019.

[21] BattulgaDavaasambuu, TumneeTelmuun, Dominik Sasko, Yu Keping, and ShirmenSodbileg, "A Novel Anchor Selection Scheme for Distributed Mobility Management", Computer Science, vol.no. 22, no. 1, 2021.

[22] Yong-hwan Kim, Hyun-kyo Lim, Kyoung-han Kim, and Youn-Hee Han, "A SDNbased distributed mobility management in LTE/EPC network", The Journal of Supercomputing, vol.no. 73, no. 7, pp. 2919-2933, 2017 
[23] Cherry Ye Aung, Boon Chong Seet, Mingyang Zhang, Ling Fu Xie, and Peter Han Joo Chong, "A review of group mobility models for mobile ad hoc networks", Wireless Personal Communications, vol.no.85, no. 3, pp. 1317-1331, 2015.

[24] Mani Shekhar Gupta, and KrishanKumar,"Application aware networks' resource selection decision making technique using group mobility in vehicular cognitive radio networks", Vehicular Communications, vol.no.26, 100263, 2020

[25] TugceBilen, BerkCanberk, and Kaushik R. Chowdhury, "Handover management in software-defined ultra-dense 5G networks", IEEE Network, vol.no.31, no. 4, pp. 49-55, 2017.

[26] Mohammed Balfaqih, Zain Balfaqih, Vladimir Shepelev, Soltan A. Alharbi, and Waheb A. Jabbar, "An analytical framework for distributed and centralized mobility management protocols", Journal of Ambient Intelligence and Humanized Computing, pp.1-13, 2020.

[27] Subodh Kumar,, G. S. Agrawal, and Sudhir Kumar Sharma, "Impact of Mobility on MANETs Routing Protocols Using Group Mobility Model", International Journal of Wireless and Microwave Technologies, vol. no. 7, no. 2, pp. 1-12, 2017. 
Figures

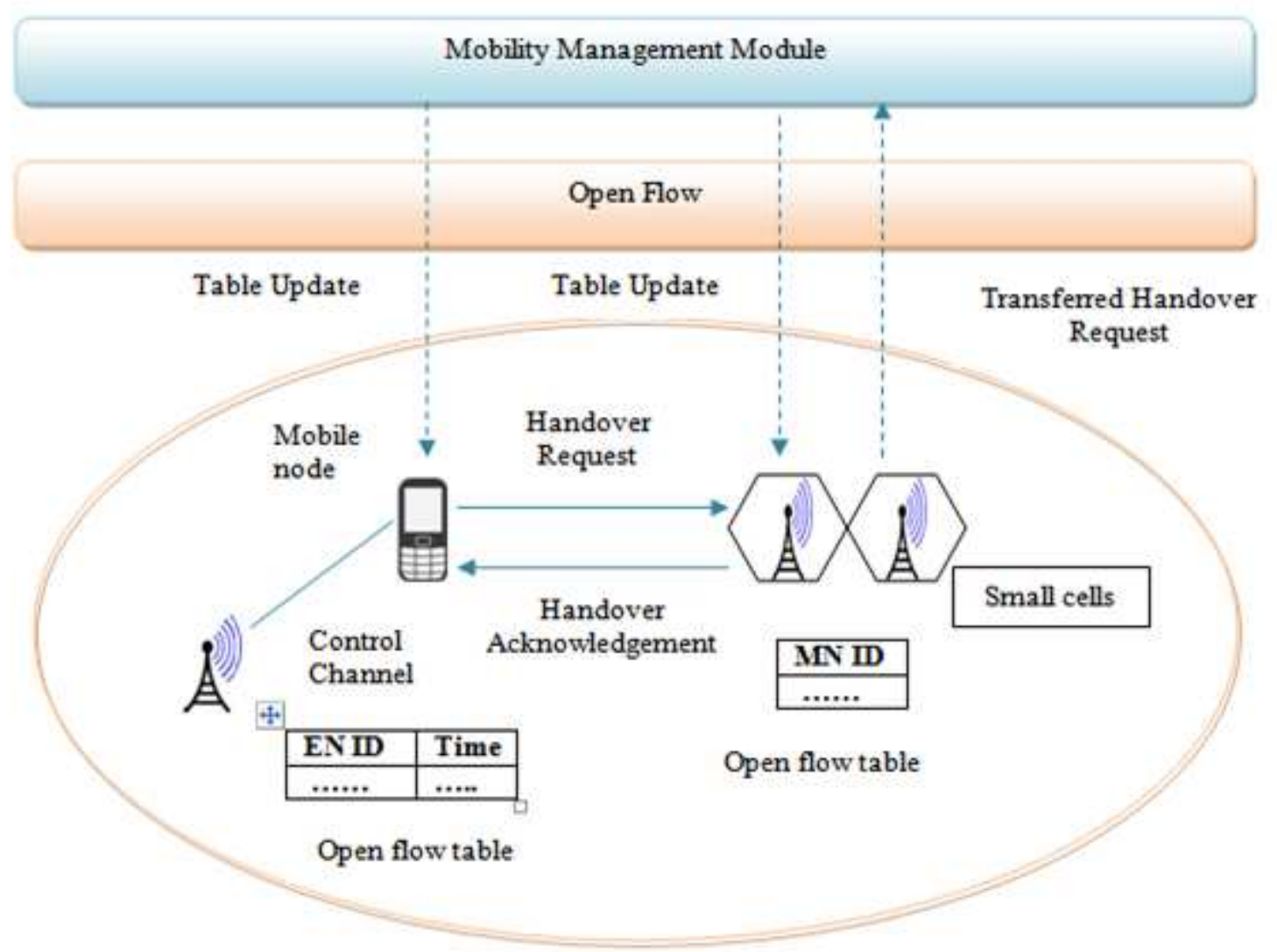

Figure 1

The proposed network architecture

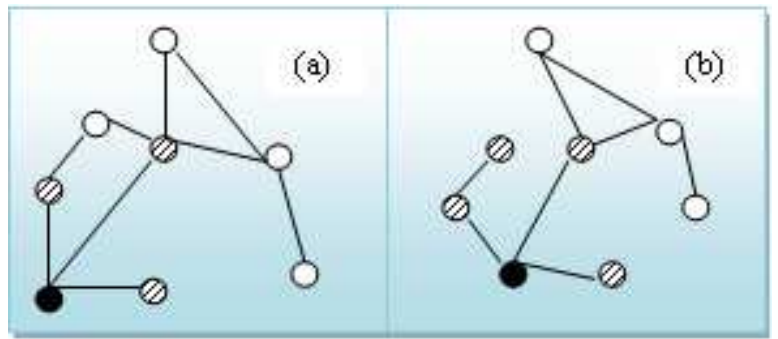

Figure 2

Example of the function of the SDN - GMM MoMo mobility model for a group of 8 nodes. 


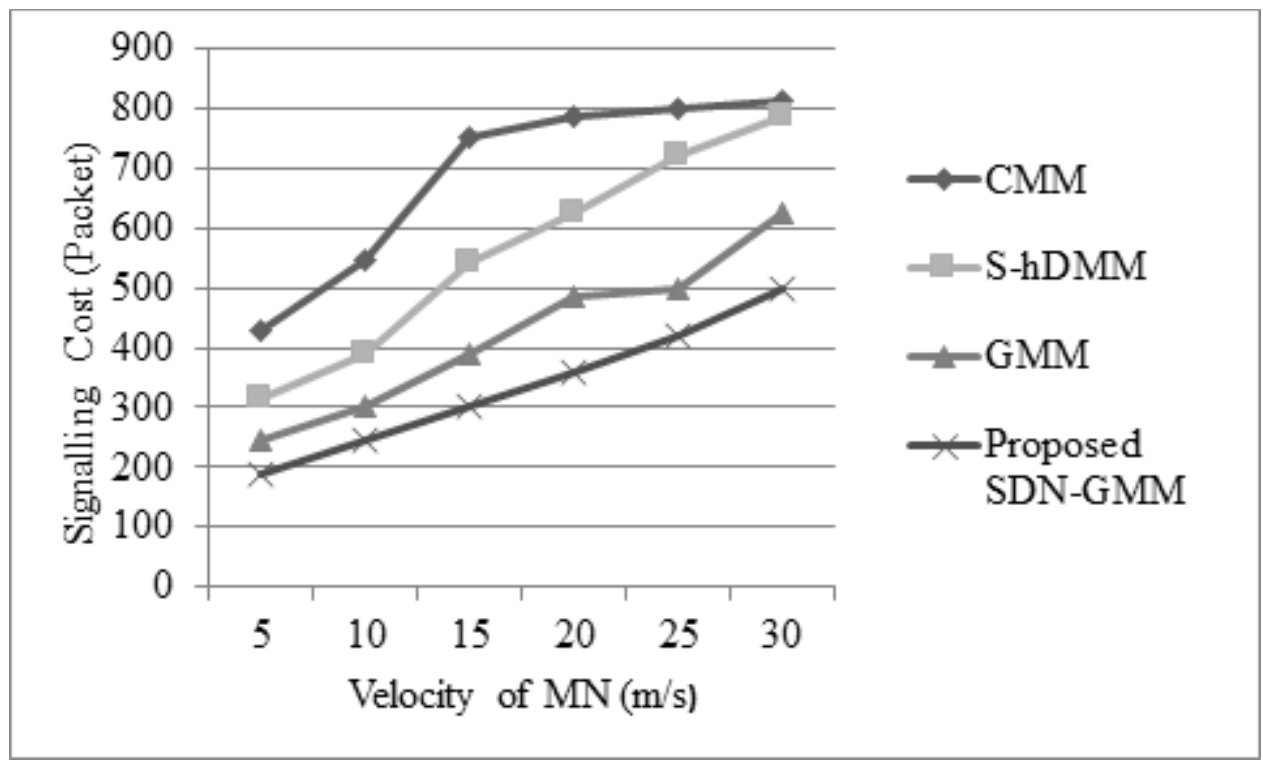

Figure 3

Handover signaling cost versus velocity of MNs

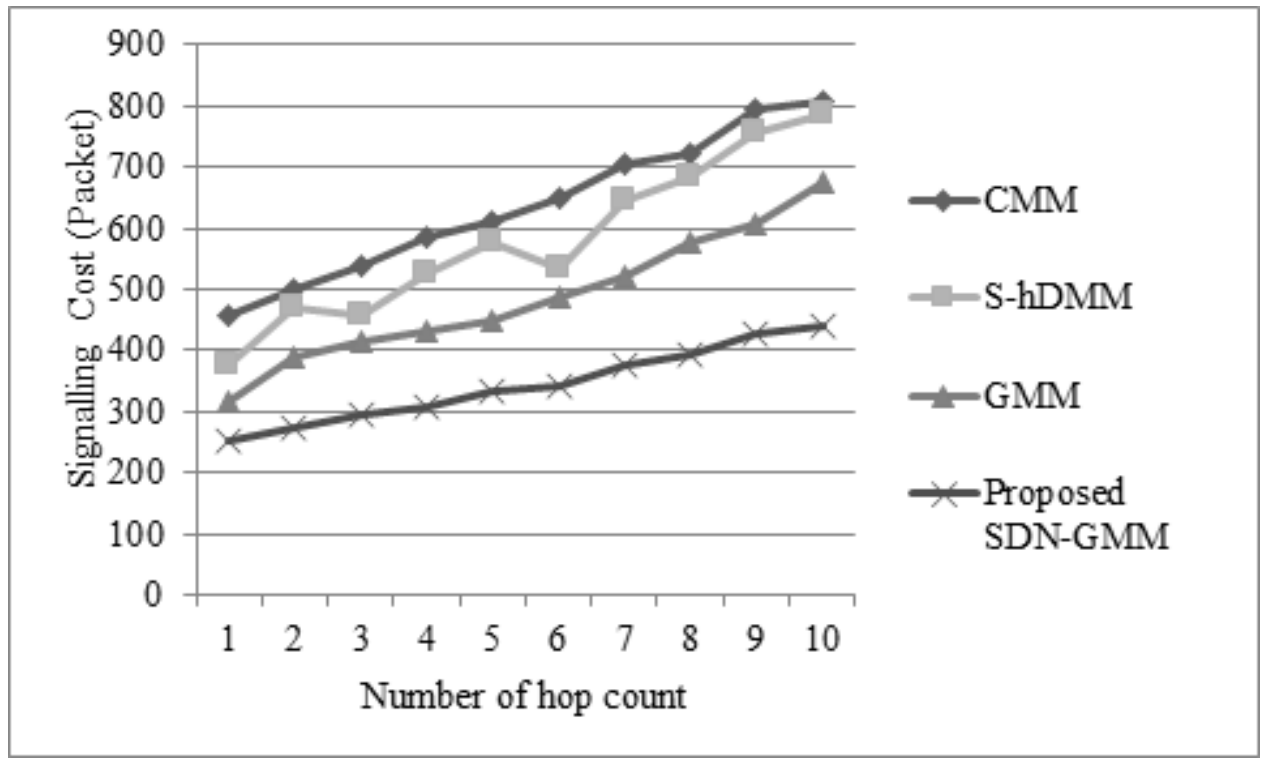

Figure 4

Handover signaling cost versus number of hop count 


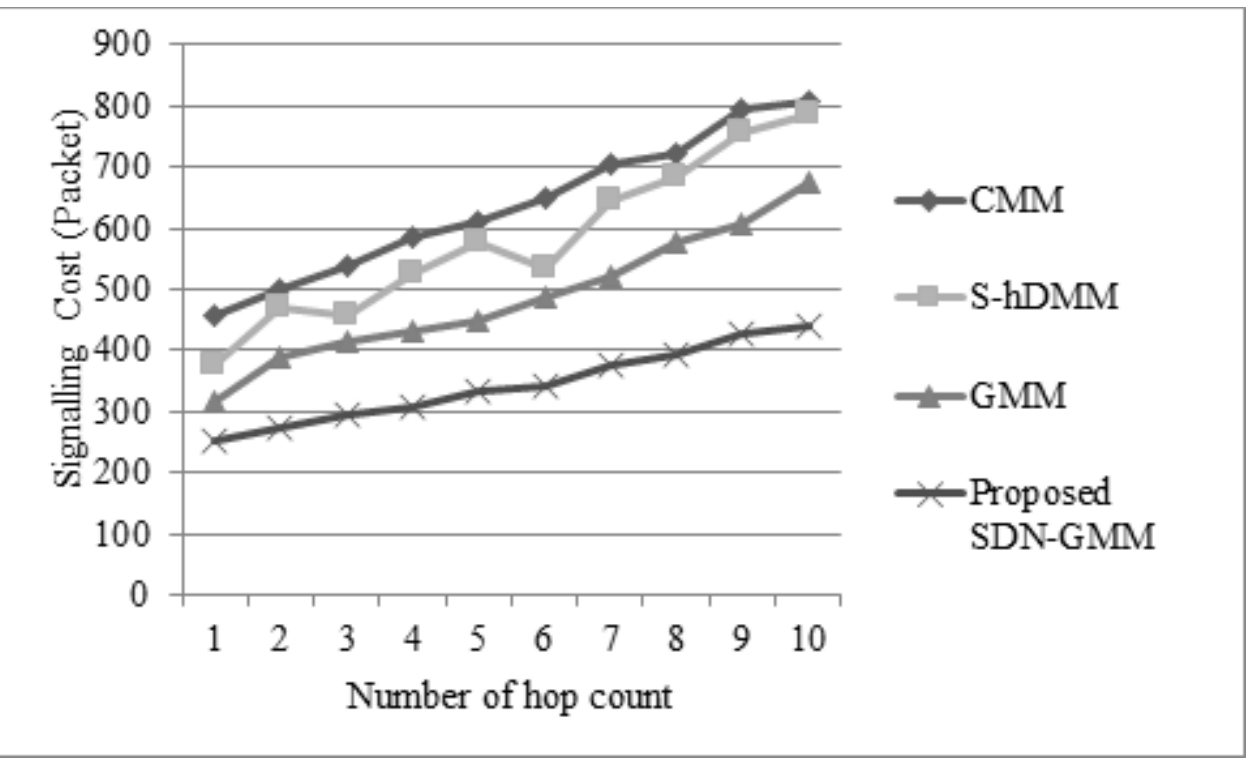

Figure 5

Handover signaling cost versus radius of cells

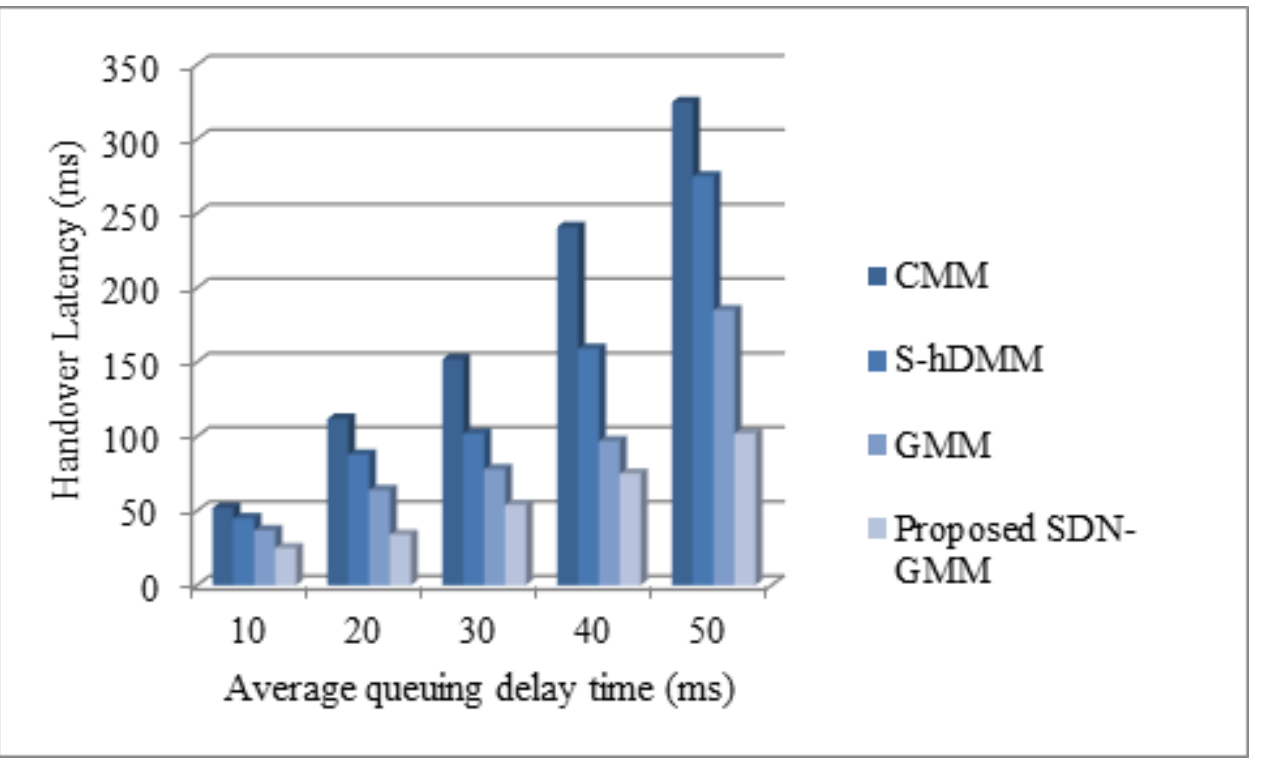

Figure 6

Handover delay versus average queuing delay 


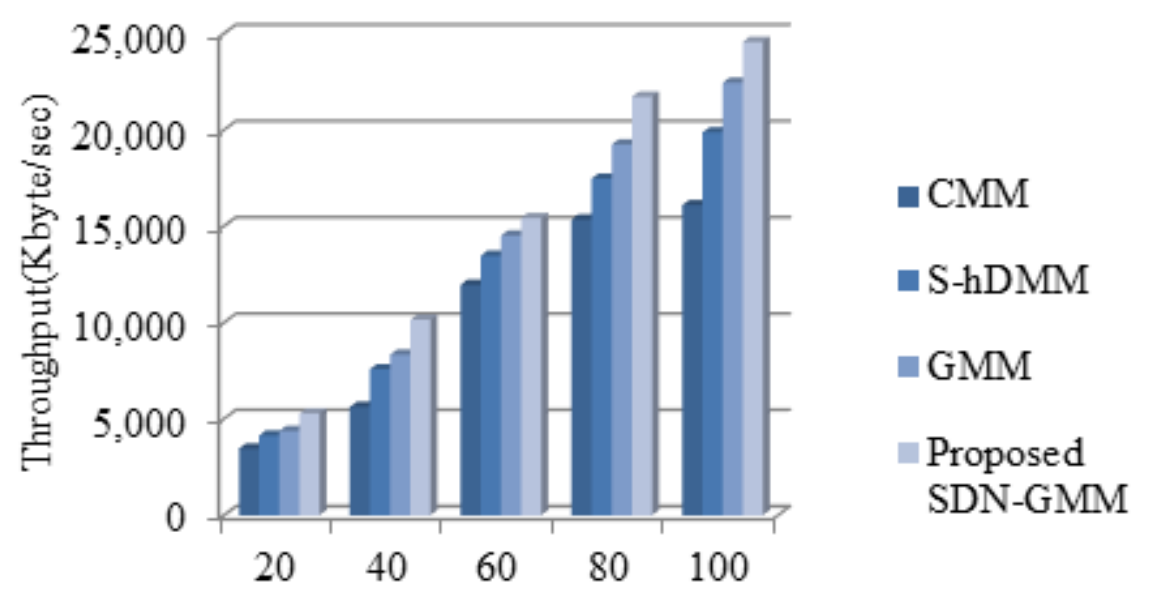

Average no. of transmitted Packets

Figure 7

Throughput versus count of packets in a session

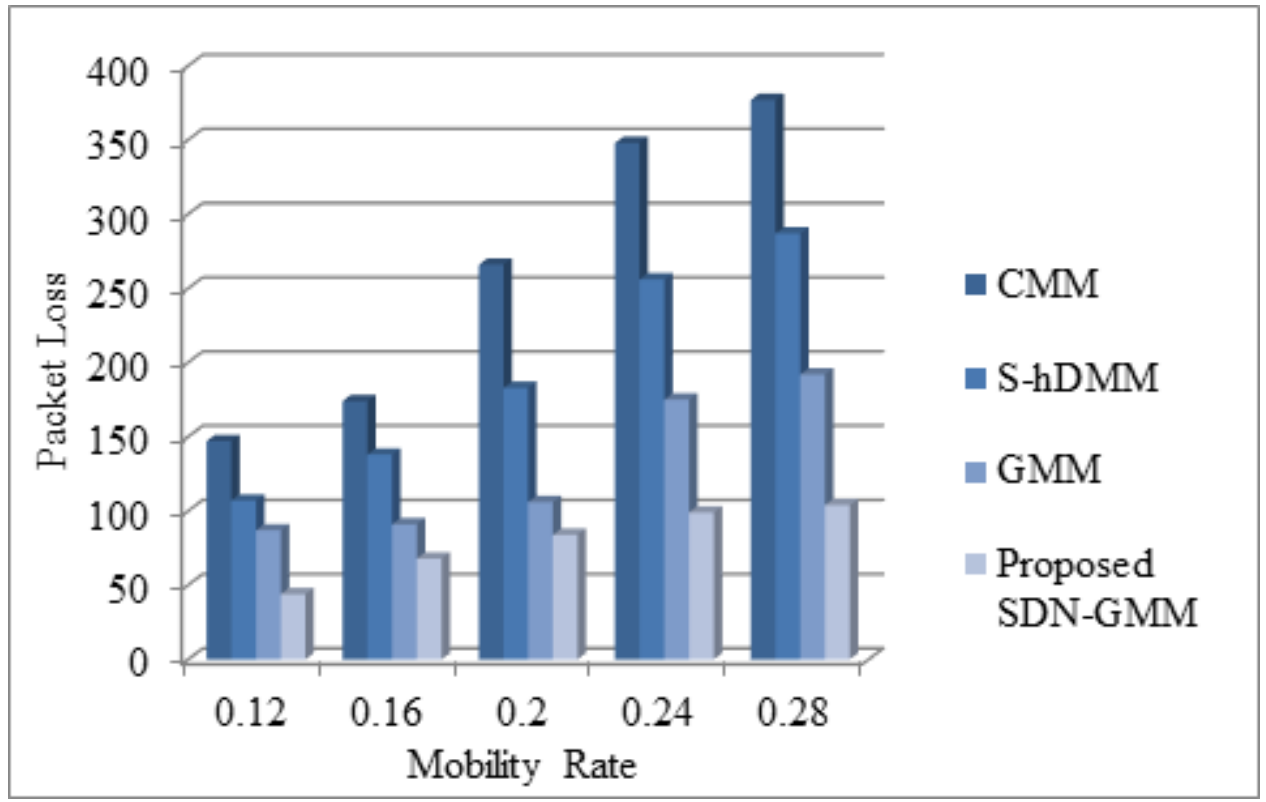

Figure 8

Packet loss versus mobility rate 